MOL2NET 2021, International Conference on Multidisciplinary Sciences, 7th edition

sciforum

NIXMSM-07: North-Ibero-American Exp., Model., and Simul. Methods

Workshop, Valencia, Bilbao, Spain-Miami, USA, 2021

\title{
Significant Study of Qualitative Research In Health Care
}

\author{
Ajit Singh ${ }^{a}$ \\ ${ }^{a}$ Department of Computer Science, Patna Women's College, Patna, India
}

\begin{abstract}
Scientific research is based upon finding a solution to a particular problem one can identify. There are various methods of formulating a research design for the study. Two broad approaches of data collection and interpretation in research are qualitative and quantitative research. The elementary method of conducting research was quantitative, but recently, qualitative method of research has also gained momentum among researchers.
\end{abstract}

Qualitative research focuses in understanding a research query as a humanistic or idealistic approach. Though quantitative approach is a more reliable method as it is based upon numeric and methods that can be made objectively and propagated by other researchers. Qualitative method is used to understand people's beliefs, experiences, attitudes, behavior, and interactions. It generates non-numerical data. The integration of qualitative research into intervention studies is a research strategy that is gaining increased attention across disciplines. Although once viewed as philosophically incongruent with experimental research, qualitative research is now recognized for its ability to add a new dimension to interventional studies that cannot be obtained through measurement of variables alone.[1] Qualitative research was initially used in psychological studies when researchers found it tedious to evaluate human behavior in numeric. Since then, qualitative research is used in other research fields as well. In clinical research, qualitative approach can help view the data more extensively. It strengthens clinical trials by enhancing user involvement in it.

In this paper, the nature of qualitative research, Qualitative research designs, Methods of collecting qualitative data, Handling qualitative research data, Analysing qualitative results and Presenting the result.

Keywords: Qualitative Research, Design, Methods, Analysis, Techniques, Result 


\section{Introduction}

A starting point in trying to understand the collection of information for research purposes is that there are broadly two approaches: quantitative research and qualitative research. Early forms of research originated in the natural sciences such as biology, chemistry, physics, geology etc. and was concerned with investigating things which we could observe and measure in some way. Such observations and measurements can be made objectively and repeated by other researchers. This process is referred to as "quantitative" research.

Much later, along came researchers working in the social sciences: psychology, sociology, anthropology etc. They were interested in studying human behaviour and the social world inhabited by human beings. They found increasing difficulty in trying to explain human behaviour in simply measurable terms. Measurements tell us how often or how many people behave in a certain way but they do not adequately answer the question "why?". Research which attempts to increase our understanding of why things are the way they are in our social world and why people act the ways they do is "qualitative" research.

The purpose of this resource paper is to enable researchers with little or no previous experience of research to gain a basic understanding of qualitative research and the potential for this type of research in primary research area.

The paper begins with a general introduction into the nature of qualitative research. This includes identification of the strengths and weaknesses of qualitative research in a brief comparison with quantitative research. This is followed by short descriptions of the main qualitative approaches and ways of collecting information. Clear and practical guidance is provided on techniques for analysing and presenting information.

\section{The nature of qualitative research}

Qualitative research is concerned with developing explanations of social phenomena. That is to say, it aims to help us to understand the world in which we live and why things are the way they are. It is concerned with the social aspects of our world and seeks to answer questions about:

Why people behave the way they do

How opinions and attitudes are formed

How people are affected by the events that go on around them

How and why cultures have developed in the way they have

The differences between social groups

Qualitative research is concerned with finding the answers to questions which begin with: why? how? in what way? Quantitative research, on the other hand, is more concerned with questions about: how much? how many? how often? to what extent? Further features of qualitative research and how it differs from quantitative research are listed below.

Qualitative research is concerned with the opinions, experiences and feelings of individuals producing subjective data.

Qualitative research describes social phenomena as they occur naturally. No attempt is made to manipulate the situation under study as is the case with experimental quantitative research.

Understanding of a situation is gained through an holistic perspective. Quantitative 
research depends on the ability to identify a set of variables.

Data are used to develop concepts and theories that help us to understand the social world. This is an inductive approach to the development of theory. Quantitative research is deductive in that it tests theories which have already been proposed

Qualitative data are collected through direct encounters with individuals, through one to one interviews or group interviews or by observation. Data collection is time consuming.

The intensive and time consuming nature of data collection necessitates the use of small samples.

Different sampling techniques are used. In quantitative research, sampling seeks to demonstrate representativeness of findings through random selection of subjects. Qualitative sampling techniques are concerned with seeking information from specific groups and subgroups in the population.

Criteria used to assess reliability and validity differ from those used in quantitative research

A review of textbooks reveals a variety of terms used to describe the nature of qualitative and quantitative research. Some of the common terms are listed in Table 1.

Qualitative Research Quantitative Research

$\begin{array}{ll}\text { Subjective } & \text { Objective } \\ \text { Holistic } & \text { Reductionist } \\ \text { Phenomenological } & \text { Scientific } \\ \text { Anti positivist } & \text { Positivist } \\ \text { Descriptive } & \text { Experimental } \\ \text { Naturalistic } & \text { Contrived } \\ \text { Inductive } & \text { Deductive }\end{array}$

Table 1: Comparison of qualitative and quantitative research terms.

Each of the various features of qualitative research may be viewed as a strength or as a weakness. This depends on the original purpose of the research. For example, one common criticism levied at qualitative research is that the results of a study may not be generalisable to a larger population because the sample group was small and the subjects were not chosen randomly. But the original research question may have sought insight into a specific subgroup of the population, not the general population because the subgroup is "special" or different from the general population and that specialness is the focus of the research. The small sample may have been necessary because very few subjects were available such as is the case with some ethnic groups or patient groups suffering from a rare condition. In such studies, generalisibility of the findings to a wider, more diverse population is not an aim.

\section{Qualitative research designs}

In this section, four major types of qualitative research design are outlined. They are:

phenomenology

ethnography

grounded theory 
case study

Another common research design is the survey. Surveys can be either qualitative or quantitative in their approach to data collection.

\title{
Phenomenology
}

The terminology used by different authors can be very confusing and the use of the term phenomenology is one example. In Table 1 in Section 1 of this paper, phenomenology was listed as one of the terms used to describe qualitative research generally. However, it is also used to describe a particular type of qualitative research.

Phenomenology literally means the study of phenomena. It is a way of describing something that exists as part of the world in which we live. Phenomena may be events, situations, experiences or concepts. We are surrounded by many phenomena, which we are aware of but not fully understand. Our lack of understanding of these phenomena may exist because the phenomenon has not been overtly described and explained or our understanding of the impact it makes may be unclear. For example, we know that lots of people are carers. But what does "caring" actually mean and what is it like to be a carer?

Back pain is another example. Correlation studies may tell us about the types of people who experience back pain and the apparent causes. Randomised controlled trials of drugs compare the effectiveness of one analgesia against another. But what is it actually like to live with back pain? What are the effects on peoples' lives? What problems does it cause? A phenomenological study might explore, for example, the effect that back pain has on sufferers' relationships with other people by describing the strain it can cause in marriages or the effect on children of having a disabled parent.

Phenomenological research begins with the acknowledgement that there is a gap in our understanding and that clarification or illumination will be of benefit. Phenomenological research will not necessarily provide definitive explanations but it does raise awareness and increases insight.

\section{Ethnography}

Ethnography has a background in anthropology. The term means "portrait of a people" and it is a methodology for descriptive studies of cultures and peoples. The cultural parameter is that the people under investigation have something in common. Examples of parameters include:

\author{
geographical - a particular region or country \\ religious \\ tribal \\ shared experience
}

In research area settings, researchers may choose an ethnographic approach because the cultural parameter is suspected of affecting the population's response to care or treatment. For example, cultural rules about contact between males and females may contribute to reluctance of women from an Asian subgroup to take up cervical screening. Ethnography 
helps research area professionals to develop cultural awareness and sensitivity and enhances the provision and quality of care for people from all cultures.

Ethnographic studies entail extensive fieldwork by the researcher. Data collection techniques include both formal and informal interviewing, often interviewing individuals on several occasions, and participant observation. Because of this, ethnography is extremely time consuming as it involves the researcher spending long periods of time in the field.

Analysis of data adopts an "emic" approach. This means that the researcher attempts to interpret data from the perspective of the population under study. The results are expressed as though they were being expressed by the subjects themselves, often using local language and terminology to describe phenomena. For example, a researcher may explore behaviour which we traditionally in the westernised medical world would describe as mental illness. However, within the population under study, the behaviour may not be characterised as illness but as something else - as evidence that the individual is "blessed" or "gifted" in some way.

Ethnographic research can be problematic when researchers are not sufficiently familiar with the social mores of the people being studied or with their language. Interpretation from an "etic" perspective - an outsider perspective - may be a misinterpretation causing confusion. For this reason, the ethnographic researcher usually returns to the field to check his interpretations with informants thereby validating the data before presenting the findings.

\section{Grounded theory}

This methodology originated with Glaser and Strauss and their work on the interactions between research area professionals and dying patients. The main feature is the development of new theory through the collection and analysis of data about a phenomenon. It goes beyond phenomenology because the explanations that emerge are genuinely new knowledge and are used to develop new theories about a phenomenon. In research area settings, the new theories can be applied enabling us to approach existing problems in a new way. For example, our approaches to health promotion or the provision of care.

One example of grounded theory with which many of us are familiar is theory about the grief process. Researchers observed that people who were bereaved progressed through a series of stages and that each stage was characterised by certain responses: denial, anger, acceptance and resolution. This is not a new phenomenon, people have going through these stages for as long as society has existed, but the research formally acknowledged and described the experience. Now we use our knowledge of the grief process, new knowledge derived from grounded theory, to understand the experience of bereavement and to help the bereaved to come to terms with their loss. We recognise when a person is having difficulty coming to terms with loss because we use the knowledge to recognise signs of "abnormal" grief and can offer help.

Various data collection techniques are used to develop grounded theory, particularly interviews and observation although literature review and relevant documentary analysis make important contributions. A key feature of grounded theory is the simultaneous collection and analysis of data using a process known as constant comparative analysis. In this process, data are transcribed and examined for content immediately following data collection. Ideas which emerge from the analysis are included in data collection when the researcher next enters the field. For this reason, a researcher collecting data through semi 
structured interviews may gradually develop an interview schedule in the latter stages of a research project which looks very different to the original schedule used in the first interview.

New theory begins its conception as the researcher recognises new ideas and themes emerging from what people have said or from events which have been observed. Memos form in the researcher's consciousness as raw data is reviewed. Hypotheses about the relationship between various ideas or categories are tested out and constructs formed leading to new concepts or understandings. In this sense the theory is "grounded" in the data.

As in phenomenology where there are concepts of which we are aware but do not fully understand, there are aspects of research area which might be informed by the development of new theory. One example is spirituality. In any holistic programme of care research area professionals may talk about the need to meet the "spiritual needs" of patients. However, we understand very little of what this means. At first sight, spiritual needs might be interpreted as referring to religious beliefs but many people would say that spiritual needs are more than this. It may be an individual's sense of well being, happiness or peace of mind. Grounded theory research could provide research area professionals with a better framework for providing truly holistic care.

\section{Case study}

Like surveys, case study research is one of those research approaches which can take a qualitative or quantitative stance. In this resource paper, the qualitative approach to case study is described wherein the value of case study relates to the in depth analysis of a single or small number of units. Case study research is used to describe an entity that forms a single unit such as a person, an organisation or an institution. Some research studies describe a series of cases.

Case study research ranges in complexity. The most simple is an illustrative description of a single event or occurrence. More complex is the analysis of a social situation over a period of time. The most complex is the extended case study which traces events involving the same actors over a period of time enabling the analysis to reflect changes and adjustments.

As a research design, the case study claims to offer a richness and depth of information not usually offered by other methods. By attempting to capture as many variables as possible, case studies can identify how a complex set of circumstances come together to produce a particular manifestation. It is a highly versatile research method and employs any and all methods of data collection from testing to interviewing.

Case study research in research area has a range of uses. For example, a case study may be conducted of the development of a new service such as a hospital discharge liaison scheme jointly run by health and social services in one locality. Another example of the case study approach would be to describe and analyse organisational change in the planning, purchasing or delivery of health services as in Total Purchasing pilot projects. One of the most common uses of the case study is the evaluation of a particular care approach. For example, an outreach teenage health service set up as an alternative to general practice based teenage clinics might be evaluated in terms of input, impact on the health of teenagers locally and the development of collaborative links with other groups involved in promoting teenage health.

One of the criticisms aimed at case study research is that the case under study is not necessarily representative of similar cases and therefore the results of the research are not generalisable. 
This is a misunderstanding of the purpose of case study research which is to describe that particular case in detail. It is particularistic and contextual. For example, the usefulness of an outreach teenage health service would be determined by a number of local factors and an evaluation of the service would take those factors into account. If the service works well it does not automatically mean that the service would work equally well in another part of the country but the lack of generalisability does not lessen the value of the service in the area where it is based. Generalisability is not normally as issue for the researcher who is involved in studying a specific situation. It is an issue for the readers who want to know whether the findings can be applied elsewhere. It is the readers who must decide whether or not the case being described is sufficiently representative or similar to their own local situation.

\section{Methods of collecting qualitative data}

Qualitative approaches to data collection usually involve direct interaction with individuals on a one to one basis or in a group setting. Data collection methods are time consuming and consequently data is collected from smaller numbers of people than would usually be the case in quantitative approaches such as the questionnaire survey. The benefits of using these approaches include richness of data and deeper insight into the phenomena under study.

Unlike quantitative data, raw qualitative data cannot be analysed statistically. The data from qualitative studies often derives from face-to-face interviews, focus groups or observation and so tends to be time consuming to collect. Samples are usually smaller than with quantitative studies and are often locally based. Data analysis is also time consuming and consequently expensive.

The main methods of collecting qualitative data are:

individual interviews

focus groups

observation

This section provides an overview of these methods.

\section{The interview}

Interviews can be highly structured, semi structured or unstructured. Structured interviews consist of the interviewer asking each respondent the same questions in the same way. A tightly structured schedule of questions is used, very much like a questionnaire. The questions may even be phrased in such a way that a limited range of responses can be elicited. For example: "Do you think that health services in this area are excellent, good, average or poor? Bearing in mind the cost of conducting a series of one to one interviews, the researcher planning to use structured interviews should carefully consider the information could be more efficiently collected using questionnaires.

Semi structured interviews (sometimes referred to as focused interviews) involve a series of open ended questions based on the topic areas the researcher wants to cover. The open ended nature of the question defines the topic under investigation but provides opportunities 
for both interviewer and interviewee to discuss some topics in more detail. If the interviewee has difficulty answering a question or provides only a brief response, the interviewer can use cues or prompts to encourage the interviewee to consider the question further. In a semi structured interview the interviewer also has the freedom to probe the interviewee to elaborate on the original response or to follow a line of inquiry introduced by the interviewee. An example would be:

Interviewer: "I'd like to hear your thoughts on whether changes in government policy have changed the work of the doctor in general practice. Has your work changed at all?"

Interviewee: "Absolutely! The workload has increased for a start."

Interviewer: "In what way has it increased?"

Unstructured interviews (sometimes referred to as "depth" or "in depth" interviews have very little structure at all. The interviewer goes into the interview with the aim of discussing a limited number of topics, sometimes as few as one or two, and frames the questions on the basis of the interviewee's previous response. Although only one or two topics are discussed they are covered in great detail. The interview might begin with the interviewer saying: "I'd like to hear your views on the GP role in PCTs". Subsequent questions would depend on how the interviewee responded. Unstructured interviews are exactly what they sound like - interviews where the interviewer wants to find out about a specific topic but has no structure or preconceived plan or expectation as to how they will deal with the topic. The difference with semi structured interviews is that in a semi structured interview the interviewer has a set of broad questions to ask and may also have some prompts to help the interviewee but the interviewer has the time and space to respond to the interviewees responses.

Qualitative interviews are semi structured or unstructured. If the interview schedule is too tightly structured this may not enable the phenomena under investigation to be explored in terms of either breadth or depth. Semi structured interviews tend to work well when the interviewer has already identified a number of aspects he wants to be sure of addressing. The interviewer can decide in advance what areas to cover but is open and receptive to unexpected information from the interviewee. This can be particularly important if a limited time is available for each interview and the interviewer wants to be sure that the "key issues" will be covered.

Qualitative interviews should be fairly informal. Interviewees should feel as though they are participating in a conversation or discussion rather than in a formal question and answer situation. However, achieving this informal style is dependent on careful planning and on skill in conducting the interview. More information on the skills required of the interviewer can be found in the Trent Focus Resource paper Using Interviews in a Research Project.

Semi structured interviews should not be seen as a soft option requiring little forethought. Good quality qualitative interviews are the result of rigorous preparation. The development of the interview schedule, conducting the interview and analysing the interview data all require careful consideration and preparation. These matters are discussed in the Trent Focus Resource paper: 'Using Interviews in a Research Project'. 


\section{Focus groups}

Sometimes it is preferable to collect information from groups of people rather than from a series of individuals. Focus groups can be useful to obtain certain types of information or when circumstances would make it difficult to collect information using other methods to data collection. They have been widely used in the private sector over the past few decades, particularly market research. They are being increasing used in the public sector.

Group interviews can be used when:

Limited resources prevent more than a small number of interviews being undertaken.

It is possible to identify a number of individuals who share a common factor and it is desirable to collect the views of several people within that population sub group.

Group interaction among participants has the potential for greater insights to be developed.

\section{Characteristics of a focus group}

The recommended size of a group is of $6-10$ people. Smaller than this limits the potential on the amount of collective information. More than this makes it difficult for everyone to participate and interact.

Several focus groups should be run in any research project. It would be wrong to rely on the views of just one group. The group may be subject to internal or external factors of which the investigator is unaware. This can lead to idiosyncratic results. Individual groups may not go very well: the members may be reluctant to participate or not interact well with each other and limited insight will be gained. Sufficient groups should be run to provide adequate breadth and depth of information but a small number of groups may achieve this, as few as three or four. There is no upper limit on the number of focus group interviews that could be held although this will be limited by resources.

The members of each focus group should have something in common, characteristics which are important to the topic of investigation. For example, they may all be members of the same profession or they may work in the same team. They may all be patients at a practice or have experienced a similar health problem or be receiving similar treatment. Participants might or might not know each other. There are advantages and disadvantages to both.

Following on from (3), focus groups are usually specially convened groups. It may be necessary or even desirable to use pre formed groups but difficulties may occur. This is usually due to the pre existing purpose of the group which can lead to the group having a particular perspective or bias which limits their potential for providing information. For example, pressure groups or groups with some political basis.

Qualitative information is collected which makes use of participants' feelings, perceptions and opinions. Just as in individual interviews data collection and analysis is time consuming. 
Using qualitative approaches requires certain skills. The researchers require a range of skills: groups skills in facilitating and moderating, listening, observing and analysing.

\section{Handling qualitative research data}

Interviewers have a choice of whether to take notes of responses during the interview or to tape record the interview. The latter is preferable for a number of reasons. The interviewer can concentrate on listening and responding to the interviewee and is not distracted by trying to write down what has been said. The discussion flows because the interviewer does not have to write down the response to one question before moving on to the next. In note taking there is an increased risk of interviewer bias because the interviewer is likely to make notes of the comments which make immediate sense or are perceived as being directly relevant or particularly interesting. Tape recording ensures that the whole interview is captured and provides complete data for analysis so cues that were missed the first time can be recognised when listening to the recording. Lastly, interviewees may feel inhibited if the interviewer suddenly starts to scribble: they may wonder why what they have just said was of particular interest.

The ideal tape recorder is small, unobtrusive and produces good quality recording. An in built microphone makes the participants less self-conscious. An auto reverse facility means that the tape will automatically "turn itself over" if the interview lasts longer than the recording time available on one side of the tape: this prevents an interruption in the flow of conversation. A tape recorder with a counter facility can be useful when analysing the taped data (see below).

\section{Transcribing qualitative data}

Transcribing is the procedure for producing a written version of the interview. It is a full "script" of the interview. Transcribing is a time consuming process. The estimated ratio of time required for transcribing interviews is about 5:1. This means that it can take two and a half hours or more to transcribe a thirty minute interview. It also produces a lot of written text as one interview can run to up to 20 pages.

It may not be essential to transcribe every interview. It is possible to use a technique known as tape analysis which means taking notes from a playback of the tape recorded interview. If tape analysis is used the counter facility can be useful because the researcher can listen to the tape and make a note of the sections which contain particularly useful information and key quotations and return to these sections of the tape for fuller analysis. However, the previously mentioned problems of bias can occur if inexperienced qualitative researchers attempt tape analysis. It is certainly preferable to produce full transcripts of the first few interview data. Once the researcher becomes familiar with the key messages emerging from the data tape analysis may be possible.

The researcher should consider the question "who should do the transcribing? If the research is funded or supported by an employer there may be resources to pay an audio typist. This is usually more cost effective than a research area professional who will take longer and is 
more highly paid. However, if the transcriber is unfamiliar with the terminology or language contained in the interviews this can lead to mistakes or prolong the transcribing time.

Good quality transcribing is not simply transferring words from the tape to the page. When people are in conversation only a small proportion of the message is communicated in the actual words used. A larger proportion is transmitted in the way people speak. Tone and inflection are good indicators of a

whole range of feelings and meanings. When transcribing, consideration should be given to how these feelings and meanings can be communicated on paper by using punctuation marks, and techniques such as upper case lettering, underlining and emboldening. Take the phrase "this was good". These three words can be said in a variety of ways and mean something different in every case.

"He was ALRIGHT" (He was alright, I liked him)

"HE was alright" (He was alright but I wasn't so keen on the others)

"He WAS alright" (He used to be but he isn't now)

"He was alright?" (Well you might think so but I don't)

By listening and noting the intensity and feeling in the interviewee's voice it is possible to detect the following:

Positive / negative continuum:

Certainty / uncertainty:

Enthusiasm / reluctance:

Whether something was seen as good or bad. How sure the interviewee was about what he said. How happy or supportive the interviewee was about the topic being discussed.

Another procedure sometimes adopted when interviews are used in qualitative research is constant comparative analysis. This is a process whereby data collection and data analysis occur on an ongoing basis. The researcher conducts the first interview which may be unstructured or semi structured. The interview is transcribed and analysed as soon as possible, certainly before the next interview takes place, and any interesting findings are incorporated into the next interview. The process is repeated with each interview. When using this procedure it is quite possible that the initial interviews in a research projects are very different to the later interviews as the interview schedule has been continuously informed and revised by informants.

More information on interviewing can be found in the Trent Focus Resource paper Using interviews in a research project.

\section{Analysing qualitative data}

Analysis of data in a research project involves summarising the mass of data collected and presenting the results in a way that communicates the most important features. In quantitative research analysis involves things like the frequencies of variables, differences between variables, statistical tests designed to estimate the significance of the results and 
the probability that they did not occur by chance. All this is done basically by counting how often something appears in the data and comparing one measurement with others. At the end of the analysis, not only do we have a mass of results but we also have what we might call "the big picture", the major findings.

In qualitative research we are also interested in discovering the big picture but use different techniques to find it. As in quantitative research, there may be some data which are measurable but for the most part we are interesting in using the data to describe a phenomenon, to articulate what it means and to understand it.

The basic process of analysing quantitative and qualitative data is the same. We start by labelling or coding every item of information so that we can recognise differences and similarities between all the different items. Imagine a questionnaire which has been used to collect quantitative information about why patients go to the health centre. The questionnaire might include a question like "why did you last visit the health centre?" Respondents have a choice of answers and tick the appropriate box. The researcher pre codes the responses as follows:

\begin{tabular}{|l|l|}
\hline I felt ill & $=1$ \\
\hline To attend a health screening clinic & $=2$ \\
\hline To get a repeat prescription & $=3$ \\
\hline $\begin{array}{l}\text { A chiropody/physiotherapy } \\
\text { appointment }\end{array}$ & $=4$ \\
\hline \begin{tabular}{l} 
I needed a form signing \\
\hline
\end{tabular} & $=5$ \\
\hline
\end{tabular}

The responses from all the questionnaires can be entered into a computer and the researcher can easily count up how many people answered the question in a given way - how many people went to the health centre because they felt ill, how many went to attend a health screening clinic and so on. Another question asks whether the respondent is male (coded as ' 1 ' ) or female (coded as ' 2 '). Responses to this question can be considered in light of responses to the previous question by telling the computer to cross tabulate responses. In this way it is possible to quickly tell, for example, how many men went to the doctor for health screening versus how many women.

Coding qualitative data requires different techniques. If, for example, the researcher has used a qualitative approach to explore patients' expectations of the health centre by interviewing patients, he will have a transcript of the interview with each patient, not a questionnaire. The researcher reads through the transcript and, at some point, find reference to why the interviewee last visited the health centre. The qualitative researcher has no system for precoding so needs a method of identifying and labelling (coding) items of data which appear in the text of a transcript so that all the items of data in one interview can be compared with data collected from other interviewees. This requires a process called content analysis and the basic procedure is described below. The procedure is the same whether the qualitative data has been collected through interviews, focus groups, observation or documentary analysis since it is concerned with analysing text. 
Content analysis is a procedure for the categorisation of verbal or behavioural data, for purposes of classification, summarisation and tabulation. The content can be analysed on two levels. The basic level of analysis is a descriptive account of the data: this is what was actually said with nothing read into it and nothing assumed about it. Some texts refer to this as the manifest level or type of analysis. The higher level of analysis is interpretative: it is concerned with what was meant by the response, what was inferred or implied. It is sometimes called the latent level of analysis.

Content analysis involves coding and classifying data. Some authors refer to this as categorising or indexing. The basic idea is to identify from the transcripts the extracts of data that are informative in some way and to sort out the important messages hidden in the mass of each interview.

The procedure involves a series of steps. These are listed as follows:

Take a copy of the transcript and read through it. When you see something that contains apparently interesting or relevant information, make a brief note in the margin about the nature of the information you have noticed.

Look through your margin notes and make a list of the different types of information you have found. If the transcript was typed using a word processor, a quicker way of doing this will be to highlight each item of data, copy it and paste it onto a list (make sure you keep an original copy of the whole transcript in your file!)

You now have a list of items excerpted from the text. Read through the list of data items and categorise each item in a way that describes what it is about. You will find yourself using some of the categories several times because several items of data refer to the same topic. However, at this stage go for as many categories as you need and don't put something into the same category as a previous item of data if you even suspect that you may have identified a new category. The number of categories can be reduced later.

Now look at the list of categories you have identified from the transcript and consider whether some of the categories may be linked in some way. If so, you could list them as major categories and the original, smaller categories as minor categories. Some textbooks refer to these major categories as themes.

Look through the list of minor and major categories of data. As you do so, compare and contrast the various categories. You may find that you change your mind about some of the minor categories. As you start to develop "the big picture" you may perceive some items of data differently and see them as "fitting" better into an alternative category. Sometimes, an item seems to belong in two categories. If so, list it under both.

Move on to the next transcript and repeat the process from stages 1 - 5 . As you work through the second and subsequent transcripts you will continue to identify new categories of information but you will increasingly find that you recognise an item of data as belonging to a previously identified category. Eventually you will run out of new categories and find that all the items of relevant and interesting information can be accommodated in the existing categories.

At this stage some researchers like to colour code their categories and use a different coloured highlighter pen for each category to highlight items of data in the transcripts. This is a good idea as it makes recognition of data easier when reviewing the transcripts 
at a later stage. However, be aware that you could change your mind later about an item of data and want to move it to a different category. Always keep clean copies of transcripts so that you can go over it with a different coloured pen.

Collect together all the extracts from the transcribed interviews that you have put into one category because they appeared to bear some relationship to each other. Examine each of the extracts in turn. Do they belong together or are there any extracts that now look as though they don't fit and really belong in a different category?

When all the relevant transcript data has been sorted into minor and major categories, look again at the data contained in each category. As you review the data within the system of categorisation you have developed you may decide to move some items of data from one category to another. Or you may decide that information is in the right category, the "right place", in that it fits together, but the terms used to name or describe the category is inaccurate.

Once you have sorted out all the categories and are sure that all the items of data are in the right category, look at the range of categories to see whether two or more categories seem to fit together. If so they may form a major theme in your research.

Go back to the original copies of the transcripts, the ones where you made your initial notes in the margins. Look at any text that you did not highlight at all because it did not appear relevant at the time. Now you have the themes, major categories and minor categories clearly sorted, consider whether any of the previously excluded data is relevant and should be included in your results.

This process may appear confusing at first. It seems as though the qualitative researcher keeps changing his mind about data and has difficulty deciding what data belongs where. To some extent this is true. The process of content analysis involves continually revisiting the data and reviewing the categorisation of data until the researcher is sure that the themes and categories used to summarise and describe the findings are a truthful and accurate reflection of the data.

\section{Computerised data analysis}

Software paperages have been available for a long time to make analysis of quantitative data quicker and easier. In recent years software has been developed which can help with the analysis of qualitative data. An increasing range of paperages is available, each one with different features and some are more popular with new researchers than others. Essentially they work on the principle of assist with the process of categorisation then collecting together items of information which appear to match under the given categories. The paperages, if properly used, can save the researcher a great deal of time but a fair amount of human input is still required to identify and check categorisation.

Some of the most well known software paperages are listed below.

ATLAS/ti

NVivo

NUD*IST

It is possible to access further information on many of these paperages by looking on the 
Internet. Some companies have their own web sites. There are also a number of networks aimed at qualitative researchers who can be accessed via the Internet. One such network is CAQDAS The site address is http://www.soc.surrey.ac.uk/caqdas

\section{Tape analysis}

It is advisable, if at all possible, to analyse qualitative data using transcribed records of data. If transcripts of recorded interviews are not available it is possible to carry out tape analysis. This involves replaying the tape recording of an interview and making notes of relevant and interesting data rather than full transcripts. It is much less time consuming than transcript analysis but it has a number of disadvantages. The procedure is open to researcher bias as the researcher is likely to make notes of information that is immediately recognisable as useful and potentially relevant information may be overlooked. The quality of the analysis may also lack depth and comprehensiveness. This can compromise the accuracy of data and therefore the reliability and validity.

\section{Presenting the results of qualitative research}

Qualitative data has several features to take into consideration when planning the presentation of findings. The data are subjective, interpretative, descriptive, holistic and copious and it can be difficult to know where or how to start. A good starting point is to look at the themes and categories which have emerged and to use these to structure the results section of the research report.

This structure can be set out at the beginning, either as a list or in diagrammatic form. The themes are then presented in sections with the categories as sub sections. In this way, the categories of data are used to construct a case that the themes are the main findings of the study. Further "evidence" to support the findings is provided by using direct quotations from respondents. Key quotations are selected to illustrate the meaning of the data. Consider this example. It shows the part of the structure of themes and categories which emerged from an investigation into the need for an outreach teenage health clinic.

\begin{tabular}{|c|c|c|}
\hline Themes & Major categories & Minor categories \\
\hline \multirow[t]{3}{*}{$\begin{array}{l}\text { 1. Health issues for young } \\
\text { people }\end{array}$} & Sexual health & $\begin{array}{l}\text { safe sex, pregnancy, sexual } \\
\text { behaviour, sexuality }\end{array}$ \\
\hline & Drugs & smoking, alcohol, illicit drugs \\
\hline & Mental health & $\begin{array}{l}\text { mental health problems, } \\
\text { relationships,self esteem, stress }\end{array}$ \\
\hline \multirow[t]{2}{*}{\begin{tabular}{|l}
$\begin{array}{l}\text { 2. Barriers to accessing } \\
\text { services }\end{array}$ \\
\end{tabular}} & Lack of knowledge & $\begin{array}{l}\text { services available, } \\
\text { understanding, perceptions }\end{array}$ \\
\hline & Attitudes & $\begin{array}{l}\text { own beliefs, peer pressure, } \\
\text { expectations of staff }\end{array}$ \\
\hline \multirow[t]{2}{*}{ 3. Incentives to use services } & Availability & time, venue \\
\hline & Approachability & staff attributes, environment \\
\hline
\end{tabular}


A presentation of these findings would describe what was meant by "health issues" in general for young people. This would be followed by identification and description of each of the broad categories of health issue - sexual health, drugs and mental health. Each category of health issue describes how a range of topics is included in this category (the minor categories). Quotations are extracted from the transcripts of interviews with young people to illustrate why or how this is a health issue.

Quotations should be used because they are good examples of what people have said specifically about the category being described. A range of quotations should be selected to illustrate such features as: the strength of opinion or belief; similarities between respondents; differences between respondents; the breadth of ideas.

As the researcher works through the different categories, the links between categories should be made to demonstrate how the themes emerged and how conclusions about the findings were drawn. Many of the quotations will "speak for themselves" as they are examples of the manifest level of analysis - what people actually said. However, as previously mentioned in Section 4, some analysis of data is carried out at the latent or interpretative level which involves extracting the meaning of what was said. Careful selection of quotations will demonstrate the reliability and validity of the data analysis.

Some qualitative data can be dealt with in a quantitative way. If an idea appears in the data frequently it may be feasible to measure how often it appears. In the example of the teenage outreach service, it may be possible to say how many respondents identified sexual health as a health issue, how many identified drugs and how many identified mental health. By counting the number of respondents who mention contraception as opposed to the number who mention safe sex it may appear that contraception is a greater concern than safe sex for young people. It may be feasible or even desirable to present some of the results quantitatively using tables and figures. Using qualitative and quantitative techniques for analysis of data can strengthen the analysis. 


\section{Conclusion}

The purpose of this paper was to provide to enable readers with no previous knowledge to understand, at a basic level, how qualitative research is undertaken. By describing the nature of qualitative research and the different research designs, Sections 1 and 2 started to demonstrate the potential for qualitative approaches to be used to investigate research problems in primary research area settings.

Four type of qualitative research designs approaches have been outlined. They do not form an exhaustive list and some research methods can be applied with either a qualitative or a quantitative orientation. The language of qualitative research is not easy for the novice researcher to understand as it often refers to abstract ideas. This is not helped by diversity in the use of terms among qualitative writers.

The differences between the various qualitative research designs can be difficult to understand at first. The differences are quite subtle and are mainly concerned with the original research question, the people or situations being studied and the way the data is analysed, interpreted and presented. Readers of this resource paper should not worry if they do not fully understand the difference between phenomenology and grounded theory or between ethnography and case study at this stage in their reading. The main purpose of this section is to familiarise the reader with the notion that there are different qualitative methodologies and what the terms mean.

By raising some of the issues involved in collecting and analysing qualitative data in Sections 3,4 and 5, novice researchers can start to appreciate the complexity of qualitative research. The paper is designed as a starting point for anyone contemplating qualitative research but further reading is necessary to understand these complexities more fully. A selection of the more widely available texts is listed at the end of this paper. 


\section{References}

Bryman A, Burgess R (Eds) (1993) Analysing Qualitative Data. London, Routledge.

Burnard P (1991) A method of analysing interview transcripts in qualitative research. Nurse Education Today 11, 461-466.

Burnard $P$ (1994) Using a database programme to handle qualitative data. Nurse Education Today. 14(3) 228-231.

Carter Y, Thomas C (Eds) (1997) Research Methods in Primary Care. Oxon, Radcliffe Medical Press.

Cobb AK, Hagemaster JN (1987) Ten criteria for evaluating qualitative research proposals. Journal of Nursing Education 26(4) 138-143.

Cooke M (1992) Computer analyses of qualitative data: a literature review of current issues. Australian Journal of Advanced Nursing. 10 (1) 10-13.

Glaser BG, Strauss AL (1967) The Discovery of Grounded Theory. Chicago, Aldine.

Hammersley M, Atkinson P (1989) Ethnography: Principles in Practice. London, Routledge.

Kreuger RA (1994) Focus Groups: A Practical Guide For Applied Research (2nd Edition). London, Sage.

Leininger MM (1985) Qualitative Research Methods in Nursing. Grune and Stratton.

Maykut P, Morehouse R (1994) Beginning Qualitative Research: a Philosophical and Practical Guide. London, Falmer.

Mays N, Pope C (Eds) (1996) Qualitative Research in research area. London, BMJ Publishing Group.

Miles M, Huberman A (1994) Qualitative Data Analysis. Thousand Oaks, Sage.

Silverman D (1993) Interpreting Qualitative Data: Methods For Analysing Talk, Text and Interaction. London, Sage.

Sorrell JM, Redmond GM (1995) Interviews in qualitative nursing research: differing approaches for ethnographic and phenomenological studies. Journal of Advance Nursing $21(6)$ 1117-1122.

Stake RE (1995) The Art of Case Study Research Thousand Oaks, Sage.

Strauss A, Corbin J (1990) Basics of Qualitative Research: Grounded Theory Procedures and Techniques. London, Sage.

Yin RK (1994) Case Study Research: Design and Methods (2nd Edition) Newbury Park, Sage. 